$\mathrm{J}$ o u r n a l of

Mathematics

and Applications

JMA No 43, pp 99-112 (2020)

\title{
On Nonlinear Fractional Neutral Differential Equation with the $\psi$-Caputo Fractional Derivative
}

\author{
Tamer Nabil
}

\begin{abstract}
In this article, the solvability of fractional neutral differential equation involving $\psi$-Caputo fractional operator is considered using a Krasnoselskii's fixed point approach. Also, we establish the uniqueness of the solution under certain conditions. Ulam stabilities for the proposed problem are discussed. Finally, examples are displayed to aid the applicability of the theory results.
\end{abstract}

AMS Subject Classification: 47H10, 34K37.

Keywords and Phrases: Krasnoselskii's fixed point theory; $\psi$-Caputo operator; Neutral differential equation; Ulam stability; Existence of solution.

\section{Introduction}

Fractional calculus is strong tool of mathematical analysis that studies derivatives and integrals of fractional order. Fractional differential equations (FDE's, for short) are used in many fields of engineering and sciences such as dynamical of biological systems [12], economy [33], theory of control [7], automatic systems [36], signal processing [11], hydro-mechanics and non-linear elasticity [14, 32].

Various real life problems can be modeled as differential equation. The study of existence of solution of these differential equation is interest object of mathematical analysis. The fixed point theorems are powerful technique to obtain the existence of solution of these problem. There are many of fixed point theorems can be applied to obtain the solution of mathematical models [24, 25]. Krasnoselskii's and Banach fixed point theorems play an important role to obtain the existence of solution of a lot of mathematical problems [35].

COPYRIGHT (c) by Publishing House of Rzeszów University of Technology P.O. Box 85, 35-959 Rzeszów, Poland 
In 1940, Ulam purposed new role of the stability analysis of the solutions for functional equations [34]. In the next year, Hyer [15] considered another type of stability in the Banach space which was more generalized than the kind of Ulam stability and applied this stability approach to obtain the stability certain conditions of some functional equations. After that, Rassias [27] considered another approach of stability, this approach is more improved than Hyers stabitity. Rassias used this approach to study stability of FDE's [16, 28].

Recently, many research articles study the Ulam stabilities, see $[21,20,13,10,8$, $2,22,3,19,17,18,30]$. In 2011, Ardjouni and Djoudi [6] studied the stability for neutral ordinary differential equations via fixed points. In 2019, Akbulut and Tunc [1], established the stability of solutions of neutral ordinary differential equations with multiple time delay. In the same year, Niazi [26], discussed Ulam stabilities for nonlinear fractional neutral differential equations in Caputo sense via Picard operator.

There are many definitions are used to define the fractional derivative such as Riemann-Liouville, Caputo, Erdélyi-Kober and Hadamard [23]. More recently, Almeida [4] considers new investigation of the fractional operator and called it $\psi$-Caputo derivative. This new approach is more generalized than RiemannLiouville, Caputo, Erdélyi-Kober and Hadamard derivative operator approaches. After one year, Almeida et al.[5] investigated the uniqueness of solution of initial value problem (I.V.P, for short) of FDE in $\psi$-Caputo sense. delay

In this paper, we discuss the existence and uniqueness of the following FDE with

$$
\left\{\begin{array}{l}
{ }^{*} D_{0^{+}}^{\alpha, \psi}[x(t)-H(t, x(t-\vartheta(t)))]=F(x(t), x(t-\vartheta(t))) \\
\alpha \in(0,1], t \in I=[0,1] ; \\
\text { subject to I.V. } \\
x(t)=\sigma(t), t \in[\rho, 0] ;
\end{array}\right.
$$

where ${ }^{*} D^{\alpha, \psi}$ is $\psi$-Caputo derivative operator, the delay $\rho=\inf \{t-\vartheta(t): t \in$ $[0,1]\} \leq 0, \vartheta: \mathbb{R}^{+} \rightarrow \mathbb{R}^{+}$and $\sigma:[\rho, 0] \rightarrow \mathbb{R}$.

\section{Preliminaries}

In this section, we consider some facts and basic results. We recall the following definition [3].

Definition 2.1. Let $C([\rho, 1], \mathbb{R})$ be the vectorial space of all continuous functions $u:[\rho, 1] \rightarrow \mathbb{R}$. Clearly, $C([\rho, 1], \mathbb{R})$ is a complete normed space with the norm, $\|u\|=\max _{t \in[\rho, 1]}|u(t)|$. Therefore, $C^{n}([\rho, 1], \mathbb{R}), n \in \mathbb{N}$, be the vectorial space of all $n$-times continuous and differentiable functions from $[\rho, 1]$ to $\mathbb{R}$. $[4,5]$.

Next, we recall the definitions of $\psi$-fractional integral and derivative operators

Definition 2.2. Let $I=[0,1]$ and $\psi \in C^{n}(I, \mathbb{R})$, be an increasing functions such that $\psi^{\prime}(t) \neq 0$ for all $t \in I$. Consider an integrable function $u: I \rightarrow \mathbb{R}$. The 
$\psi$-Riemann-Liouville fractional integral of order $\alpha>0, \alpha \in \mathbb{R}$ of the function $u$ is defined as

$$
J_{0^{+}}^{\alpha, \psi} u(t)=\frac{1}{\Gamma(\alpha)} \int_{0}^{t} \psi^{\prime}(\zeta)(\psi(t)-\psi(\zeta))^{\alpha-1} u(\zeta) d \zeta,
$$

and the $\psi$-Riemann-Liouville fractional derivative of order $\alpha>0, \alpha \in \mathbb{R}$ of the function $u$ is defined as

$$
D_{0^{+}}^{\alpha, \psi} u(t)=\frac{1}{\Gamma(n-\alpha)}\left(\frac{1}{\psi^{\prime}(t)} \frac{d}{d t}\right)^{n} \int_{0}^{t} \psi^{\prime}(\zeta)(\psi(t)-\psi(\zeta))^{n-\alpha-1} u(\zeta) d \zeta,
$$

where $n=[\alpha]+1$ and $[\alpha]$ denotes the integral part of $\alpha$.

Definition 2.3. Let $\psi \in C^{n}(I, \mathbb{R})$, be an increasing function such that $\psi^{\prime}(t) \neq 0$ for all $t \in I$. Consider an integrable function $u: I \rightarrow \mathbb{R}$. The $\psi$-Caputo fractional derivative of order $\alpha>0, \alpha \in \mathbb{R}$ of the function $u$ is defined as

$$
{ }^{*} D_{0^{+}}^{\alpha, \psi} u(t)=D_{0^{+}}^{\alpha, \psi}\left[u(t)-\sum_{k=0}^{n-1} \frac{u_{\psi}^{[k]}(0)}{k !}(\psi(t)-\psi(0))^{k}\right],
$$

where $n=[\alpha]+1,[\alpha]$ denotes the integral part of $\alpha$ and $u_{\psi}^{[k]}(t)=\left(\frac{1}{\psi^{\prime}(t)} \frac{d}{d t}\right)^{k} u(t)$.

We recall the following Lemma which was given in [5].

Lemma 2.4. Suppose that $u: I \rightarrow \mathbb{R}$, then

(1) If $u \in C(I, \mathbb{R})$, then ${ }^{*} D_{0^{+}}^{\alpha, \psi} J_{0^{+}}^{\alpha, \psi} u(t)=u(t)$.

(2) If $u \in C^{n}(I, \mathbb{R})$, then

$$
J_{0^{+}}^{\alpha, \psi *} D_{0^{+}}^{\alpha, \psi} u(t)=u(t)-\sum_{k=0}^{n-1} \frac{u_{\psi}^{[k]}(0)}{k !}(\psi(t)-\psi(0))^{k} .
$$

Now we recall Krasnoselskii's fixed point theorem which was given in [31].

Theorem 2.5. (Krasnoselskii's fixed point theorem) Let $\Upsilon$ be a Banach space. Suppose that $\Omega \quad(\Omega \neq \emptyset)$ be a convex, bounded and closed subset of $\Upsilon$. Consider $\mathcal{T}_{1}: \Upsilon \rightarrow \Upsilon$ and $\mathcal{T}_{2}: \Omega \rightarrow \Upsilon$ are such that

(1) $\mathcal{T}_{1}$ be a contraction.

(2) $\mathcal{T}_{2}$ is completely continuous.

(3) $x=\mathcal{T}_{1} x+\mathcal{T}_{2} y \Rightarrow x \in \Omega$ for all $y \in \Omega$.

Then, there exists $x^{*} \in \Omega$ such that $x^{*}=\mathcal{T}_{1} x^{*}+\mathcal{T}_{2} x^{*}$.

Now, we recall the definitions of these types of Ulam stability. For more details, see [29].

Definition 2.6. The Eq.(1) is said to be Ulam-Hyers stable (UHS for short) if, there exists $\lambda \in \mathbb{R}^{+}$such that for every $\varepsilon>0$ and each $u \in C([\rho, 1], \mathbb{R})$ solution of the inequality

$$
\left|{ }^{*} D_{0^{+}}^{\alpha, \psi}[u(t)-H(t, u(t-\vartheta(t)))]-F(u(t), u(t-\vartheta(t)))\right| \leq \varepsilon \quad, t \in I,
$$


there exists a unique solution $x \in C([\rho, 1], \mathbb{R})$ of Eq.(1) such that

$$
|u(t)-x(t)| \leq \lambda \varepsilon \quad, \forall t \in[\rho, 1] .
$$

Definition 2.7. The Eq.(1) is said to be generalized Ulam-Hyers stable (GUHS for short) if, there exists $\varphi \in C([\rho, 1], \mathbb{R}), \varphi(0)=0$, such that for every $\varepsilon>0$ and each $u \in C([\rho, 1], \mathbb{R})$ solution of the inequality

$$
\left|{ }^{*} D_{0^{+}}^{\alpha, \psi}[u(t)-H(t, u(t-\vartheta(t)))]-F(u(t), u(t-\vartheta(t)))\right| \leq \varepsilon \quad, t \in I,
$$

there exists a unique solution $x \in C([\rho, 1], \mathbb{R})$ of Eq.(1) such that

$$
|u(t)-x(t)| \leq \varphi(\varepsilon) \quad, \forall t \in[\rho, 1] .
$$

Definition 2.8. The Eq.(1) is called Ulam-Hyers-Rassias stable (UHRS for short) w.r.t $\varphi \in C([\rho, 1], \mathbb{R})$, if there exists $\kappa_{\varphi} \in \mathbb{R}^{+}$such that for every $\varepsilon>0$ and each $u \in C([\rho, 1], \mathbb{R})$ solution of the inequality

$$
\left|{ }^{*} D_{0^{+}}^{\alpha, \psi}[u(t)-H(t, u(t-\vartheta(t)))]-F(u(t), u(t-\vartheta(t)))\right| \leq \varepsilon \varphi(t) \quad, t \in I,
$$

there exists a unique solution $x \in C([\rho, 1], \mathbb{R})$ of Eq.(1) such that

$$
|u(t)-x(t)| \leq \kappa_{\varphi} \varepsilon \varphi(t) \quad, \forall t \in[\rho, 1] .
$$

Definition 2.9. The Eq.(1) is said to be generalized Ulam-Hyers-Rassias stable (GUHRS for short) w.r.t $\varphi \in C([\rho, 1], \mathbb{R})$, if there exists $\kappa_{\varphi} \in \mathbb{R}^{+}$such that for each $u \in C([\rho, 1], \mathbb{R})$ solution of the inequalities

$$
\left|{ }^{*} D_{0^{+}}^{\alpha, \psi}[u(t)-H(t, u(t-\vartheta(t)))]-F(u(t), u(t-\vartheta(t)))\right| \leq \varphi(t) \quad, t \in I,
$$

there exists a unique solution $u \in C([\rho, 1], \mathbb{R})$ of the Eq.(1) such that

$$
|u(t)-x(t)| \leq \kappa_{\varphi} \varphi(t) \quad, \forall t \in[\rho, t] .
$$

Let $H: I \times \mathbb{R} \rightarrow \mathbb{R}$ and $F: \mathbb{R} \times \mathbb{R} \rightarrow \mathbb{R}$. Then we study the Ulam stabilities of the following proposed problem

$$
\left\{\begin{array}{l}
{ }^{*} D_{0^{+}}^{\alpha, \psi}[x(t)-H(t, x(t-\vartheta(t)))]=F(x(t), x(t-\vartheta(t))) ; \\
\alpha \in(0,1], t \in I=[0,1] ; \\
\text { subject to initial value } \\
x(t)=\sigma(t), t \in[\rho, 0] ;
\end{array}\right.
$$

where ${ }^{*} D^{\alpha, \psi}$ is $\psi$-Caputo derivative operator, $\rho=\inf \{t-\vartheta(t): t \in[0,1]\} \leq 0$, $\vartheta: \mathbb{R}^{+} \rightarrow \mathbb{R}^{+}, \sigma:[\rho, 0] \rightarrow \mathbb{R}$ are continues and $\psi \in C^{1}(I, \mathbb{R})$ be an increasing function such that $\psi^{\prime}(t) \neq 0$ for all $t \in I$. Then, we have the following lemma [9].

Lemma 2.10. The solution of Eq.(1) is equivalent to the following nonlinear integral equation

$$
\begin{aligned}
x(t)= & \sigma(0)-H(0, \sigma(-\vartheta(0)))+H(t, x(t-\vartheta(t))) \\
& +\frac{1}{\Gamma(\alpha)} \int_{0}^{t} \psi^{\prime}(s)(\psi(t)-\psi(s))^{\alpha-1} F(x(s), x(s-\vartheta(s))) d s .
\end{aligned}
$$




\section{Existence and Uniqueness}

In this section we will obtain the existence of solution and uniqueness of the proposed neutral FDE (1). suppose that $r_{0} \in \mathbb{R}^{+}$and $\Omega=\left\{x \in C([\rho, 1], \mathbb{R}):\|x\| \leq r_{0}\right\}$. The Eq.(1) can be written as

$$
(\mathcal{T} x)(t)=\left(\mathcal{T}_{1} x\right)(t)+\left(\mathcal{T}_{2} x\right)(t)
$$

where

$$
\mathcal{T}_{1}: \Omega \rightarrow\left(C B([\rho, 1], \mathbb{R}) \quad, \quad \mathcal{T}_{2}: \Omega \rightarrow(C B([\rho, 1], \mathbb{R})\right.
$$

such that

$$
\begin{aligned}
& \left(\mathcal{T}_{1} x\right)(t)=\sigma(0)-H(0, \sigma(-\vartheta(0)))+H(t, x(t-\vartheta(t))) \\
& \left(\mathcal{T}_{2} x\right)(t)=\frac{1}{\Gamma(\alpha)} \int_{0}^{t} \psi^{\prime}(s)(\psi(t)-\psi(s))^{\alpha-1} F(x(s), x(s-\vartheta(s))) d s,
\end{aligned}
$$

where $t \in[\rho, 1]$ and $x \in C([\rho, 1], \mathbb{R})$.

We will study Eq.(1) under the following conditions:

(C1) the functions $H: I \times \mathbb{R} \rightarrow \mathbb{R}$ and $F: \mathbb{R} \times \mathbb{R} \rightarrow \mathbb{R}$ are continuous and there exist $p \in(0,1), q \in \mathbb{R}^{+}$such that

$$
\begin{gathered}
\left|H\left(t, x_{1}\right)-H\left(t, x_{2}\right)\right|<L\left|x_{1}-x_{2}\right|, \\
\left|F\left(x_{1}, x_{1}\right)-F\left(y_{1}, y_{2}\right)\right|<K \sum_{i=1}^{2}\left|x_{i}-y_{i}\right|,
\end{gathered}
$$

for all $x_{1}, x_{2}, y_{1}, y_{2} \in \mathbb{R}$, and $t \in[0,1]$;

$(\mathrm{C} 2)$ let $A^{*}=|F(0,0)|$ and $B^{*}=\max _{t \in I}|H(t, 0)|$ then

$$
|\sigma(0)-H(0, \sigma(-\vartheta(0)))|+L r_{0}+B^{*}+\frac{K r_{0}+A^{*}}{\Gamma(\alpha+1)}(\psi(1)-\psi(0))^{\alpha} \leq r_{0} .
$$

Theorem 3.1. Let the conditions (C1) and (C2) hold. Then Eq.(1) has at leat one solution in $\Omega$.

Proof. The proof is done in the following 3 steps.

Step 1. $\mathcal{T}_{1}$ is contraction.

Let $x, y \in C([\rho, 1], \mathbb{R})$ are arbitrary and $t \in I$

$$
\left|\left(\mathcal{T}_{1} x\right)(t)-\left(\mathcal{T}_{1} y\right)(t)\right| \leq L|x(t)-y(t)|,
$$

which implies that

$$
\left\|\mathcal{T}_{1} x-\mathcal{T}_{1} y\right\| \leq L\|x-y\|
$$

Thus, $\mathcal{T}_{1}$ is a contraction.

Step 2. $\mathcal{T}_{2}$ is completely continuous.

First, we will prove that $\mathcal{T}_{2}$ is continuous. Let $\left\{x_{n}\right\}$ be a sequence in $C([\rho, 1], \mathbb{R})$ such that $x_{n} \rightarrow x \in C([\rho, 1], \mathbb{R})$. Then, we get 


$$
\begin{aligned}
\left|\left(\mathcal{T}_{2} x_{n}\right)(t)-\left(\mathcal{T}_{2} x\right)(t)\right| & \leq \frac{1}{\Gamma(\alpha)} \int_{0}^{t} \psi^{\prime}(s)(\psi(t)-\psi(s))^{\alpha-1} \mid F\left(x_{n}(s), x_{n}(s-\vartheta(s))\right) \\
& -F(x(s), x(s-\vartheta(s))) \mid d s \\
& \leq \frac{K}{\Gamma(\alpha+1)}(\psi(t)-\psi(0))^{\alpha}\left\|x_{n}-x\right\| \\
& \leq \frac{K}{\Gamma(\alpha+1)}(\psi(1)-\psi(0))^{\alpha}\left\|x_{n}-x\right\| .
\end{aligned}
$$

So, we have that

$$
\left\|\mathcal{T}_{2} x_{n}-\mathcal{T}_{2} x\right\| \leq \frac{K}{\Gamma(\alpha+1)}(\psi(1)-\psi(0))^{\alpha}\left\|x_{n}-x\right\| .
$$

Thus, $\left\|\mathcal{T}_{2} x_{n}-\mathcal{T}_{2} x\right\| \rightarrow 0$ as $n \rightarrow \infty$. Hence $\mathcal{T}_{2}$ is continuous operator. Therefore, for each $x, y \in C([\rho, 1], \mathbb{R})$ and $t \in I$, we have

$$
\begin{aligned}
|F(x(t), y(t))| & \leq|F(x, y)-F(0,0)|+|F(0,0)| \\
& \leq K(\|x\|+\|y\|)+A^{*} .
\end{aligned}
$$

Therefore,

$$
\begin{aligned}
\mid\left(\mathcal{T}_{2} x\right)(t) & \left|\leq \frac{1}{\Gamma(\alpha)} \int_{0}^{t} \psi^{\prime}(s)(\psi(t)-\psi(s))^{\alpha-1}\right| F(x(s), x(s, s-\vartheta(s))) \mid d s \\
& \leq \frac{2 K+A^{*}}{\Gamma(\alpha+1)}(\psi(t)-\psi(0))^{\alpha}
\end{aligned}
$$

for all $t \in I$. Hence we have

$$
\left\|\mathcal{T}_{2} x\right\| \leq \frac{2 K+A^{*}}{\Gamma(\alpha+1)}(\psi(1)-\psi(0))^{\alpha} .
$$

Thus $\mathcal{T}_{2}$ is bounded. Furthermore, if we choose $t_{1}, t_{2} \in I$ such that $t_{1}<t_{2}$, then we get

$$
\begin{aligned}
& \left|\left(\mathcal{T}_{2} x\right)\left(t_{2}\right)-\left(\mathcal{T}_{2} x\right)\left(t_{1}\right)\right| \\
& =\mid \frac{1}{\Gamma(\alpha)} \int_{0}^{t_{2}} \psi^{\prime}(s)\left(\psi\left(t_{2}\right)-\psi(s)\right)^{\alpha-1} F(x(s), x(s-\vartheta(s))) d s \\
& \quad-\frac{1}{\Gamma(\alpha)} \int_{0}^{t_{1}} \psi^{\prime}(s)\left(\psi\left(t_{1}\right)-\psi(s)\right)^{\alpha-1} F(x(s), x(s-\vartheta(s))) d s \mid \\
& \leq \mid \frac{1}{\Gamma(\alpha)} \int_{0}^{t_{2}} \psi^{\prime}(s)\left(\psi\left(t_{2}\right)-\psi(s)\right)^{\alpha-1} F(x(s), x(s-\vartheta(s))) d s \\
& \quad-\frac{1}{\Gamma(\alpha)} \int_{0}^{t_{2}} \psi^{\prime}(s)\left(\psi\left(t_{1}\right)-\psi(s)\right)^{\alpha-1} F(x(s), x(s-\vartheta(s))) d s \mid \\
& \quad+\mid \frac{1}{\Gamma(\alpha)} \int_{0}^{t_{2}} \psi^{\prime}(s)\left(\psi\left(t_{1}\right)-\psi(s)\right)^{\alpha-1} F(x(s), x(s-\vartheta(s))) d s \\
& \quad-\frac{1}{\Gamma(\alpha)} \int_{0}^{t_{1}} \psi^{\prime}(s)\left(\psi\left(t_{1}\right)-\psi(s)\right)^{\alpha-1} F(x(s), x(s-\vartheta(s))) d s \mid \\
& \leq \frac{1}{\Gamma(\alpha)} \int_{0}^{t_{2}} \psi^{\prime}(s)\left[\left(\psi\left(t_{2}\right)-\psi(s)\right)^{\alpha-1}-\left(\psi\left(t_{1}\right)-\psi(s)\right)^{\alpha-1}\right]|F(x(s), x(s-\vartheta(s)))| d s \\
& \quad+\frac{1}{\Gamma(\alpha)} \int_{t_{1}}^{t_{2}} \psi^{\prime}(s)\left(\psi\left(t_{1}\right)-\psi(s)\right)^{\alpha-1}|F(x(s), x(s-\vartheta(s)))| d s \\
& \leq \frac{2 K r_{0}+A^{*}}{\Gamma(\alpha+1)}\left[\left(\psi\left(t_{2}\right)-\psi(0)\right)^{\alpha}-\left(\psi\left(t_{1}\right)-\psi(0)\right)^{\alpha}\right] .
\end{aligned}
$$

Since $\psi$ is continuous, then we have that $\left|\left(\mathcal{T}_{2} x\right)\left(t_{2}\right)-\left(\mathcal{T}_{2} x\right)\left(t_{1}\right)\right| \rightarrow 0$ as $t_{1} \rightarrow t_{2}$. Thus $\mathcal{T}_{2}(\Omega)$ is relatively compact. From Arzela-Ascoli-theorem, we obtain $\mathcal{T}_{2}$ is compact. Hence $\mathcal{T}_{2}$ is completely continuous. 
Step 3. Finding the fixed poind of $\mathcal{T}$.

Let $x, y \in \Omega$. We get

$$
\begin{aligned}
& \left|\left(\mathcal{T}_{1} x\right)(t)+\left(\mathcal{T}_{2} y\right)(t)\right| \\
& =\mid \sigma(0)-H(0, \sigma(-\vartheta(0)))+H(t, x(t-\vartheta(t))) \\
& \quad+\frac{1}{\Gamma(\alpha)} \int_{0}^{t} \psi^{\prime}(s)(\psi(t)-\psi(s))^{\alpha-1} F(x(s), x(s-\vartheta(s))) d s \mid \\
& \leq|\sigma(0)-H(0, \sigma(-\vartheta(0)))|+|H(t, x(t-\vartheta(t)))|+ \\
& \quad+\left|\frac{1}{\Gamma(\alpha)} \int_{0}^{t} \psi^{\prime}(s)(\psi(t)-\psi(s))^{\alpha-1} F(x(s), x(s-\vartheta(s))) d s\right| \\
& \leq|\sigma(0)-H(0, \sigma(-\vartheta(0)))|+L r_{0}+B^{*}+\frac{K r_{0}+A^{*}}{\Gamma(\alpha+1)}(\psi(t)-\psi(0))^{\alpha} \\
& \leq|\sigma(0)-H(0, \sigma(-\vartheta(0)))|+L r_{0}+B^{*}+\frac{K r_{0}+A^{*}}{\Gamma(\alpha+1)}(\psi(1)-\psi(0))^{\alpha} \\
& \leq \\
& \leq
\end{aligned}
$$

Thus, the operators $\mathcal{T}_{1}$ and $\mathcal{T}_{2}$ satisfy all conditions of Theorem 2.5. Hence there exists $x^{*} \in \Omega$ such that $x^{*}$ is solution of Eq.(1).

Theorem 3.2. Suppose that the conditions (C1) and (C2) hold. Let,

(C3) $L+\frac{2 K}{\Gamma(\alpha+1)}(\psi(1)-\psi(0))^{\alpha}<1$.

Then the Eq.(1) has unique solution.

Proof. We apply Banach contraction theorem to prove $\mathcal{T}$ has a unique fixed point. Let $x, y \in C([\rho, 1], \mathbb{R})$. Then, we have

$$
\begin{aligned}
|(\mathcal{T} x)(t)-(\mathcal{T} y)(t)| & \leq L|x(t)-y(t)|+\frac{2 K\|x-y\|}{\Gamma(\alpha)} \int_{0}^{t} \psi^{\prime}(s)(\psi(t)-\psi(s))^{\alpha-1} d s \\
& \leq L+\frac{2 K}{\Gamma(\alpha+1)}(\psi(t)-\psi(0))^{\alpha} \\
& \leq L+\frac{2 K}{\Gamma(\alpha+1)}(\psi(1)-\psi(0))^{\alpha} \\
& \leq 1
\end{aligned}
$$

Thus Eq.(1) has unique solution.

\section{Ulam Stabilities}

In this part, various Ulam stability types will be considered.

Lemma 4.1. Let $\alpha \in(0,1)$, if $z \in C([\rho, 1], \mathbb{R})$ is the solution of the inequality of definition 2.6, then $z$ is the solution of the following inequality

$$
|z(t)-N(t)| \leq\left(\frac{(\psi(1)-\psi(0))^{\alpha}}{\Gamma(\alpha+1)}\right) \varepsilon,
$$

where

$$
\begin{aligned}
N(t)= & \sigma(0)-H(0, \sigma(-\vartheta(0)))+H(t, z(t-\vartheta(t))) \\
& +\frac{1}{\Gamma(\alpha)} \int_{0}^{t} \psi^{\prime}(s)(\psi(t)-\psi(s))^{\alpha-1} F(z(s), z(s-\vartheta(s))) d s .
\end{aligned}
$$


Proof. Let $z \in C([\rho, 1], \mathbb{R})$ be any solution of the inequality of definition 2.6 , then there exists $\Theta \in C([\rho, 1], \mathbb{R})$ dependent on $z$ such that

$$
\left\{\begin{array}{l}
{ }^{*} D_{0^{+}}^{\alpha, \psi}[z(t)-H(t, z(t-\vartheta(t)))]=F(z(t), z(t-\vartheta(t)))+\Theta(t) \\
\alpha \in(0,1], t \in I=[0,1] \\
\text { subject to initial value } \\
z(t)=\sigma(t), t \in[\rho, 0]
\end{array}\right.
$$

and

$$
|\Theta(t)| \leq \varepsilon \quad, \forall t \in I .
$$

Thus, Eq.(3) is equivalent to the following equation

$$
\begin{aligned}
z(t)= & \sigma(0)-H(0, \sigma(-\vartheta(0)))+H(t, z(t-\vartheta(t))) \\
& +\frac{1}{\Gamma(\alpha)} \int_{0}^{t} \psi^{\prime}(s)(\psi(t)-\psi(s))^{\alpha-1} F(z(s), z(s-\vartheta(s))) d s \\
& +\frac{1}{\Gamma(\alpha)} \int_{0}^{t} \psi^{\prime}(s)(\psi(t)-\psi(s))^{(\alpha-1)} \Theta(s) d s .
\end{aligned}
$$

Let

$$
\begin{aligned}
N(t)= & \sigma(0)-H(0, \sigma(-\vartheta(0)))+H(t, z(t-\vartheta(t))) \\
& +\frac{1}{\Gamma(\alpha)} \int_{0}^{t} \psi^{\prime}(s)(\psi(t)-\psi(s))^{\alpha-1} F(z(s), z(s-\vartheta(s))) d s
\end{aligned}
$$

Thus, we have

$$
|z(t)-N(t)| \leq \frac{1}{\Gamma(\alpha)} \int_{0}^{t} \psi^{\prime}(s)(\psi(t)-\psi(s))^{\alpha-1}|\Theta(s)| d s \leq \frac{1}{\Gamma(\alpha+1)}(\psi(1)-\psi(0))^{\alpha} \varepsilon .
$$

Theorem 4.2. Suppose that (C1)-(C3) hold. Then the Eq.(1) is UHS and consequently GUHS.

Proof. Let $z \in C([\rho, 1], \mathbb{R})$ be a solution of the inequality of definition 2.6 and $x$ be the unique solution of Eq.(1), then we get $|N(t)| \leq \varepsilon$ for all $t \in I$ and

$$
|z(t)-x(t)| \leq|z(t)-N(t)|+|N(t)-x(t)|
$$

From Lemma 4.1, we get

$$
\begin{aligned}
|z(t)-x(t)| \leq & \left(\frac{(\psi(1)-\psi(0))^{\alpha}}{\Gamma(\alpha+1)}\right) \varepsilon_{1}+L|z(t)-x(t)| \\
& +\frac{1}{\Gamma(\alpha)} \int_{0}^{t} \psi^{\prime}(s)(\psi(t)-\psi(s))^{(\alpha-1)} 2 K|z(s)-x(s)| d s \\
\leq & \left(\frac{(\psi(1)-\psi(0))^{\alpha}}{\Gamma(\alpha+1)}\right) \varepsilon_{1}+L|z(t)-x(t)|+\frac{2 K}{\Gamma(\alpha+1)}(\psi(1)-\psi(0))^{\alpha}|z(t)-x(t)|
\end{aligned}
$$

therefore, we get

$$
\|z-x\| \leq\left(\frac{(\psi(1)-\psi(0))^{\alpha}}{\Gamma(\alpha+1)}\right) \varepsilon_{1}+\mathbb{L}\|z-x\|,
$$

where

$$
\mathbb{L}=1-\left[L+\frac{2 K}{\Gamma(\alpha+1)}(\psi(1)-\psi(0))^{\alpha}\right] .
$$


Then, we get

$$
\|z-x\| \leq \lambda \varepsilon
$$

where

$$
\lambda=\frac{\left(\frac{(\psi(1)-\psi(0))^{\alpha}}{\Gamma(\alpha+1)}\right)}{1-\mathbb{L}} .
$$

Thus the Eq.(1) is UHS. Therefore, if we put $\varphi(\varepsilon)=\lambda \varepsilon$, then we get that $\varphi(0)=0$ and

$$
\|z-x\| \leq \varphi(\varepsilon)
$$

Then, the Eq.(1) is GUHS.

Lemma 4.3. Suppose that the following condition holds:

(C4) If $\phi \in C([\rho, 1], \mathbb{R})$ is increasing, then there exists $\mu_{\phi} \in \mathbb{R}^{+}$such that for every $t \in I$, the following inequality hold

$$
{ }^{*} J_{0^{+}}^{\alpha, \psi} \phi(t) \leq \mu_{\phi} \phi(t) .
$$

If $z \in C([\rho, 1], \mathbb{R})$ is the solution of the inequality $(2)$, then $z$ is the solution of the following inequality

$$
|z(t)-N(t)| \leq \mu_{\phi}\left(\frac{(\psi(1)-\psi(0))^{\alpha}}{\Gamma(\alpha+1)}\right) \phi(t) \varepsilon
$$

Proof. From Lemma 4.1, we get

$$
|z(t)-N(t)| \leq \frac{1}{\Gamma(\alpha)} \int_{0}^{t} \psi^{\prime}(s)(\psi(t)-\psi(s))^{\alpha-1}|\Theta(s)| d s .
$$

From (C4), we have that

$$
|z(t)-N(t)| \leq \mu_{\phi}\left(\frac{(\psi(T)-\psi(0))^{\alpha}}{\Gamma(\alpha+1)}\right) \phi(t) \varepsilon .
$$

Theorem 4.4. Consider the Conditions (C1)-(C4) hold. Then the Eq.(1) is UHRS and GUHRS .

Proof. Let $z \in C([\rho, 1], \mathbb{R})$ be solution of the inequality (2) and $x$ be the unique solution of Eq.(1). From Lemma 4.3, we get

$$
\|z-x\| \leq \mu_{\phi}\left(\frac{(\psi(1)-\psi(0))^{\alpha}}{\Gamma(\alpha+1)}\right) \phi_{1}(t) \varepsilon+\mathbb{L}\|z-x\| .
$$

So, we have that

$$
\|z-x\| \leq \mu_{\phi} \lambda \phi(t) \varepsilon .
$$

Thus the Eq.(1) is UHRS. Therefore, if we put $\varepsilon=1$, then the Eq.(1) is GUHRS. 


\section{Applications}

The following examples are applications to the previous theoretical results.

Example 5.1. Consider the following $\psi$-Caputo FDE

$$
\left\{\begin{array}{l}
{ }^{*} D_{0^{+}}^{\frac{1}{3}, \psi}\left[x(t)-\frac{t e^{-t}}{10} x(t-0.1)\right]=\frac{1}{10} \tan ^{-1}(x(t))+\frac{|x(t-0.1)|}{14+|x(t-0.1)|} \\
t \in I=[0,1] \\
\text { subject to the nonlocal conditions } \\
x(t)=0.2 \quad, t \in[-0.1,0]
\end{array}\right.
$$

where $\psi(t)=\sqrt{1+t}$, for all $t \in[0,1]$. Clearly, $\psi$ is increasing on $[0,1]$ and $\psi \in$ $C^{1}([0,1], \mathbb{R})$. Therefore,

$$
H(t, x)=\frac{t e^{-t}}{10} x
$$

also

$$
F(t, x, y)=\frac{1}{10} \tan ^{-1}(x)+\frac{|y|}{14+|y|} .
$$

It is clear that, $H, F$ are continuous. Since,

$$
\begin{gathered}
\left|H\left(t, x_{1}\right)-H\left(t, x_{2}\right)\right| \leq \frac{1}{10}\left|x_{1}-x_{2}\right|, \\
\left|F\left(t, x_{1}, y_{1}\right)-F\left(t, x_{2}, y_{2}\right)\right| \leq \frac{1}{10}\left(\left|x_{1}-x_{2}\right|+\left|y_{1}-y_{2}\right|\right),
\end{gathered}
$$

for all $x, y, x_{1}, y_{1}, x_{2}, y_{2} \in \mathbb{R}$ and $t \in[0,1]$. Thus, the condition (C1) holds with

$$
L=K=\frac{1}{10},
$$

therefore

$$
A^{*}=0 \quad, \quad B^{*}=0 \quad, \quad \sigma(0)=0.2
$$

The inequality of (C2)

$$
|\sigma(0)-H(0, \sigma(-\vartheta(0)))|+L r_{0}+B^{*}+\frac{K r_{0}+A^{*}}{\Gamma(\alpha+1)}(\psi(1)-\psi(0))^{\alpha} \leq r_{0},
$$

has the following form

$$
0.2+\frac{r_{0}}{10}+\frac{r_{0}(\sqrt{2}-1)^{\frac{1}{3}}}{10 \Gamma\left(\frac{4}{3}\right)} \leq r_{0} .
$$

Hence (C2) is hold and $r_{0} \geq 0.2447531$. Similarly, we get: $L+\frac{2 K}{\Gamma(\alpha+1)}(\psi(1)-\psi(0))^{\alpha}=$ $0.11657002573<1$. Hence the condition (C3) holds. So, it is implies that, the Eq.(4) has a unique solution. Hence, the Eq.(4) is UHS, GUHS, UHRS and GUHRS. 
Example 5.2. Consider the following $\psi$-Caputo FDE

$$
\left\{\begin{array}{l}
{ }^{*} D_{0^{+}}^{\frac{1}{2}, \psi}\left[x(t)-\frac{t}{9} \sin (x(t-0.1))\right]=\frac{1}{12} x(t)+\frac{1}{10} x(t-0.1) \\
t \in I=[0,1] \\
\text { subject to the nonlocal conditions } \\
x(t)=0.2 \quad, t \in[-0.1,0]
\end{array}\right.
$$

where $\psi(t)=\frac{t^{2}+t}{2}$, for all $t \in[0,1]$. Clearly, $\psi$ is increasing on $[0,1]$ and $\psi \in$ $C^{1}([0,1], \mathbb{R})$. Therefore,

$$
H(t, x)=\frac{t}{9} \sin (x)
$$

also

$$
F(t, x, y)=\frac{1}{12} x+\frac{1}{10} y .
$$

It is clear that, $H, F$ are continuous. Since,

$$
\begin{gathered}
\left|H\left(t, x_{1}\right)-H\left(t, x_{2}\right)\right| \leq \frac{1}{9}\left|x_{1}-x_{2}\right|, \\
\left|F\left(t, x_{1}, y_{1}\right)-F\left(t, x_{2}, y_{2}\right)\right| \leq \frac{1}{10}\left(\left|x_{1}-x_{2}\right|+\left|y_{1}-y_{2}\right|\right),
\end{gathered}
$$

for all $x, y, x_{1}, y_{1}, x_{2}, y_{2} \in \mathbb{R}$ and $t \in[0,1]$. Thus, the conditions (C1) holds with

$$
L=\frac{1}{9} \quad, K=\frac{1}{10}
$$

therefore

$$
A^{*}=0, \quad B^{*}=0, \quad \sigma(0)=0.2 .
$$

The inequality of (C2)

$$
|\sigma(0)-H(0, \sigma(-\vartheta(0)))|+L r_{0}+B^{*}+\frac{K r_{0}+A^{*}}{\Gamma(\alpha+1)}(\psi(1)-\psi(0))^{\alpha} \leq r_{0},
$$

has the following form

$$
0.2+\frac{r_{0}}{10}+\frac{r_{0}}{10 \Gamma\left(\frac{3}{2}\right)} \leq r_{0}
$$

Hence (C2) is hold and $r_{0} \geq 0.25390377047$. Similarly, we get: $L+\frac{2 K}{\Gamma(\alpha+1)}(\psi(1)-$ $\psi(0))^{\alpha}=0.32471910112<1$. Hence the condition (C3) holds . So, it is implies that, the Eq.(5) has a unique solution. Hence, the Eq.(5) is UHS, GUHS, UHRS and GUHRS.

\section{References}

[1] I. Akbulut, C. Tunç, On the stability of solutions of neutral differential equations of first order, Interational J. of Mathematics and Computer Science 14 (2019) 849-866. 
[2] A. Ali, B. Samet, K. Shah, R. Khan, Existence and stability of solution of a toppled systems of differential equations non-integer order, Bound. Value Probl. 2017 (16) (2017) 1-13.

[3] Z. Ali, A. Zada, K. Shah, Ulam stability results for the solutions of nonlinear implicit fractional order differential equations, Hacet. J. Math. Stat. 48 (4) (2019) 1092-1109.

[4] R. Almeida, A Caputo fractional derivative of a function with respect to another function, Commun. Nonlinear Sci. Numer. Simul. 44 (2017) 460-481.

[5] R. Almeida, A.B. Malinowska, M.T. Monterio, Fractional differential equations with a Caputo derivative with respect to a kernel functions and their applications, Math. Method. Appl. Sci. 41 (2018) 336-352.

[6] A. Ardjouni, A. Djoudi, Stability in nonlinear neutral differential equations with variable delays using fixed point theory, Electron. J. Qual. Theory Differ. Equ. 43 (2011), 11 pp.

[7] G.M. Bahaa, Optimal control problem for variable-order fractional differential systems with time delay involving Atangana-Baleanu derivatives, Chaos, Solitons and Fractals 122 (2019) 129-142.

[8] Y. Basci, S. Ogrekçi, A. Misir, On Hyers-Ulam Stability for Fractional Differential Equations Including the New Caputo-Fabrizio Fractional Derivative, Mediterranean Journal of Mathematics (2019) 16:131.

[9] H. Boulares, A. Ardjouni, Y. Laskri, Existence and uniqueness of solutions to fractional order nonlinear neutral differential equations, Applied Mathematics E-Notes 18 (2018) 25-33.

[10] D.X. Cuong, On the Hyers-Ulam stability of Riemann-Liouville multi-order fractional differential equations, Afr. Mat. (2019) 30:1041.

[11] F. Dong, Q. Ma, Single image blind deblurring based on the fractional-order differential, Computers and Mathematics with Applications 78 (6) (2019) 19601977.

[12] M. Elettreby, A. Al-Raezah, T. Nabil, Fractional-Order Model of Two-Prey OnePredator System, Mathematical Problems in Engineering 2017 (2017), Article ID 6714538, $12 \mathrm{pp}$.

[13] Y. Guo, Xiao-Bao Shu, Y. Li, F. Xu, The existence and Hyers-Ulam stability of solution for an impulsive Riemann-Liouville fractional neutral functional stochastic differential equation with infinite delay of order $1<\alpha<2$, Bound. Value Probl. (2019) 2019:59.

[14] E. Hashemizadeh, A. Ebrahimzadeh, An efficient numerical scheme to solve fractional diffusion-wave and fractional Klein-Gordon equations in fluid mechanics, Physica A: Statistical Mechanics and its Applications 503 (2018) 1189-1203. 
[15] D.H. Hyers, On the stability of the linear functional equations, Proc. Natl. Acad. Sci. U.S.A 27 (4) (1941) 222-224.

[16] D.H. Hyers, G. Isac, T.M. Rassias, Stability of Functional Equations in Several Variables, Birkhäuser, Boston, 1998.

[17] S.-M. Jung, Hyers-Ulam-Rassias Stability of Functional Equations in Nonlinear Analysis, Springer, 2011.

[18] S.-M. Jung, D. Popa, M.Th. Rassias, On the stability of the linear functional equation in a single variable on complete metric groups, Journal of Global Optimization 59 (2014) 165-171.

[19] Pl. Kannappan, Functional Equations and Inequalities with Applications, Springer, 2009.

[20] H. Khan, T. Abdeljawad, M. Aslam, R. Khan, A. Khan, Existence of positive solution and Hyers-Ulam stability for a nonlinear singular-delay-fractional differential equation, Advances in Difference Equations, December (2019) 2019:104.

[21] A. Khan, H. Khan, J.F. Gomez-Aguilar, T. Abdeljawad, Existence and HyersUlam stability for a nonlinear singular fractional differential equations with Mittag-Leffler kernel. Chaos, Solitons and Fractals 127 (2019) 422-427.

[22] H. Khan, Y. Li, W. Chen, D. Baleanu, A. Khan, Existence theorems and HyersUlam stability for a coupled system of fractional differential equations with pLaplacian operator, Boundary Value Problems (2017) 2017:157.

[23] A.A. Kilbas, H.M. Srivastava, J.J. Trujillo, Theory and Applications of Fractional Differential Equations. North-Holland Mathematics Studies, 204. Elsevier Science B.V., Amsterdam, 2006.

[24] T. Nabil, Krasnoselskii N-Tupled Fixed Point Theorem with Applications to Fractional Nonlinear Dynamical System, Advances in Mathematical Physics 2019 (2019), Article ID 6763842, 9 pp.

[25] T. Nabil, A.H. Soliman, A Multidimensional Fixed-Point Theorem and Applications to Riemann-Liouville Fractional Differential Equations, Mathematical Problems in Engineering 2019 (2019), Article ID 3280163, 8 pp.

[26] A. Niazi, J. Wei, M. Rehman, D. Jun, Ulam-Hyers-Stability for nonlinear fractional neutral differential equations, Hacet. J. Math. Stat. 48 (2019) 157-169.

[27] T.M. Rassias, On the stability of linear mapping in Banach spaces, Proc. Amer. Math. Soc. 72 (2) (1978) 297-300.

[28] T.M. Rassias, On the stability of functional equations and a problem of Ulam, Acta. Appl. Math. 62 (2000) 23-130. 
[29] I.A. Rus, Ulam stabilites of ordinary Differential Equations in a Banach space, Carpathian J. Math. 20 (2010) 103-107.

[30] P.K. Sahoo, Pl. Kannappan, Introduction to Functional Equations, Chapman and Hall/CRC, 2017.

[31] D.R. Smart, Fixed Point Theorems, Cambridge Univ. Press, Cambridge, 1980.

[32] V.E. Tarasov, E.C. Aifantis, On fractional and fractal formulations of gradient linear and nonlinear elasticity, Acta Mechanica 230 (6) (2019) 2043-2070.

[33] D.N. Tien, Fractional stochastic differential equations with applications to finance, J. Math. Anal. Appl. 397 (2013) 338-348.

[34] S.M. Ulam, A Collection of Mathematical Problems, Interscience, New York, 1960.

[35] E. Zeidler, Nonlinear Functional Analysis and its Applications I: Fixed-Point Theorems, Springer, 1986.

[36] G.Q. Zeng, J. Chen, Y.X. Dai, L.M. Li, C.W. Zheng, M.R. Chen, Design of fractional order PID controller for automatic regulator voltage system based on multi-objective extremal optimization, Neurocomputing 160 (2015) 173-184.

\section{DOI: $10.7862 /$ rf.2020.7}

\section{Tamer Nabil}

email: t_3bdelsadek@yahoo.com

ORCID: 0000-0001-5626-4513

Department of Basic Science

Faculty of Computers and Informatics

Suez Canal University

Ismailia

EGYPT

Received 01.06.2020

Accepted 31.08.2020 